\title{
Optimized Silicon AWG With Flattened Spectral Response Using an MMI Aperture
}

\author{
Shibnath Pathak, Student Member, IEEE, Michael Vanslembrouck, Pieter Dumon, Member, IEEE, \\ Dries Van Thourhout, Member, IEEE, and Wim Bogaerts, Member, IEEE
}

\begin{abstract}
We demonstrate compact 12-channel $400 \mathrm{GHz}$ arrayed waveguide grating wavelength demultiplexers (AWG) in silicon with a flattened spectral response. Insertion loss, crosstalk and non-uniformity are $-3.29 \mathrm{~dB}, 17.0 \mathrm{~dB}$ and $1.55 \mathrm{~dB}$, respectively. The flattened spectral response is obtained by using an optimized mode shaper consisting of a multi-mode interference coupler as the input aperture of the AWG. The ratio of the $1 \mathrm{~dB}$ bandwidth to the $10 \mathrm{~dB}$ bandwidth is improved by $50 \%$, from 0.33 to 0.49 compared to a conventional AWG. The device size is only $560 \times 350 \mu \mathrm{m}^{2}$.
\end{abstract}

Index Terms-AWG, phasar (phased array), MMI, silicon-oninsulator (SOI).

\section{INTRODUCTION}

W AVELENGTH division multiplexing (WDM) is now widely used in commercial telecommunication networks. It allows to increase the capacity and provides more flexibility in complex network architectures. Since the early 1990's, Arrayed Waveguide Grating based devices (AWG) are a key component in these WDM systems, allowing for demultiplexing and routing of wavelength channels. An AWG consists of two free propagation regions (FPR) and an array of waveguides with successive increments in length [1]. The spectral response of a conventional AWG channel is Gaussian-like, but a flat spectral response is desirable, or even necessary for many WDM applications. To fulfill this requirement, one of the commonly used methods is the use of a Multi-Mode Interference Coupler (MMI) integrated with an AWG as shown in Fig. 1. This approach was first adopted by Soole et al. in lnP-based AWGs [2].

In silica-on-silicon [3] and InP [4], low contrast waveguides and the associated large bend radii result in relatively large device dimensions, which is not beneficial for the integration of many functions on a single chip. In silicon-on-insulator (SOI) the high contrast waveguides and the sharp bend radius [5], [6] allow to reduce the size of the device by several orders of magnitude [7]-[9], but make the design of such MMI couplers and the

Manuscript received May 31, 2012; revised November 07, 2012; accepted November 25, 2012. Date of publication December 03, 2012; date of current version December 17, 2012. This work was supported in part by the European Union in the framework of the FP7 Project ICT-Wadimos.

The authors are with the Department of Information Technology, Ghent University-IMEC, 9000 Ghent, Belgium (e-mail: Shibnath.Pathak@intec. Ugent.be; Michael.Vanslembrouck@intec.ugent.be; Pieter.Dumon@intec. UGent.be; dries.vanthourhout@intec.Ugent.be; Wim.Bogaerts@intec.UGent. be).

Color versions of one or more of the figures in this paper are available online at http://ieeexplore.ieee.org.

Digital Object Identifier 10.1109/JLT.2012.2231399

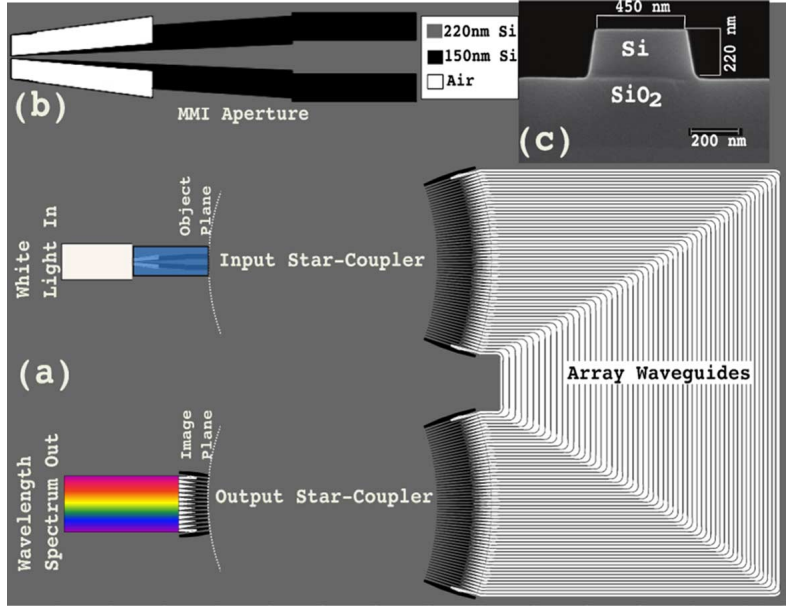

Fig. 1. (a) Schematic diagram of $12 \times 400 \mathrm{GHz}$ MMI-AWG. (b) Schematic diagram of the MMI aperture used as a input aperture of the AWG. (c) Crosssection of a silicon wire waveguide.

AWG itself difficult. The silicon waveguides used in the array are more sensitive to phase errors because of the high index contrast. As a result compact silicon AWGs typically have a higher crosstalk compared to silica-based and InP-based AWGs. However by improving the fabrication process and using wider waveguides we were able to reduce the phase errors and the associated crosstalk to an acceptable level [9].

In this paper we demonstrate an optimized design of SOI-based AWG's with an MMI at the entrance port. We optimized the MMI and the number of waveguides used in the array waveguides, and analyze the performance of the design by comparing simulation results with experiments in terms of insertion loss, crosstalk, and non-uniformity between the center and the outer channels. The aspect ratio between the $1 \mathrm{~dB}$ passband and the $10 \mathrm{~dB}$ passband of the optimized MMI-AWG is compared with that of a conventional AWG.

\section{THEORY}

The operation principle of a conventional AWG is described as follows. A light beam exiting from a suitable waveguide aperture enters into a first free propagation region (FPR) and diverges. This diverging light beam is then coupled in an array of waveguides and propagates through these waveguides to a second FPR. The optical path length difference between two successive waveguides in the array is constant and given by $\Delta L=m \cdot \lambda_{c} / N_{g}$, where $m$ is the order of the phased array, $\lambda_{c}$ is the center wavelength of the AWG, and $N_{g}$ is the group index of the waveguide. Therefore, for this center wavelength, the field distribution at the entrance of the waveguide array will be reproduced at its exit aperture. In a perfect system a perfect 


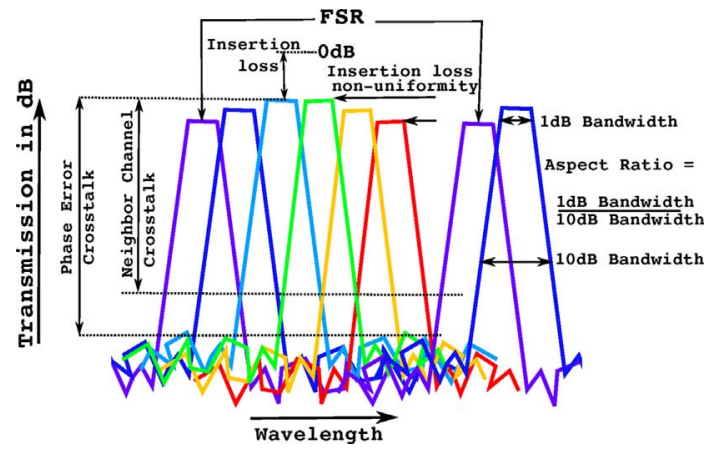

Fig. 2. Schematic representation of insertion loss, insertion loss non-uniformity, free spectral range (FSR), neighbor channel crosstalk, phase error crosstalk and passband aspect ratio.

image of the object plane can then be formed and the field distribution in the image plane will have the same amplitude and phase as the field distribution of the object plane [1]. Obviously, the imaging is never perfect because one uses a finite number of arrayed waveguides and the FPR has a finite numerical aperture.

The phase difference in the arrayed waveguides varies with wavelength because of their length difference and the wavelength-dependence of the propagation constant. This phase delay will induce a tilt in the phase fronts in the FPR and introduce a lateral shift of the image field profile. The spectral response of the AWG will therefore be determined by the overlap of this shifted field profile with the mode profile of the different output waveguide apertures located in the image plane. In most AWGs demonstrated in silicon, the geometry of the aperture at the input (object plane) and output (image plane) is identical: this results in a maximal overlap when the shifted image is perfectly aligned with one of the output apertures (i.e., at a given wavelength). However, the output power rapidly drops with a small image shift (i.e., a small wavelength shift). For many applications, such a rapid roll-off of the output power is undesirable. Therefore, we can modify the input aperture to get a better overlap for small wavelength shifts. The simplest approach is to add a multi-mode interference coupler (MMI) at the input. Fig. 1 shows the schematic layout of such an MMI-AWG. With the use of an MMI as the input for the AWG a wider twofold image can be generated in the object plane. The exact shape depends on the length, width and taper width of the MMI. With careful optimization of these parameters, we can produce a 'flat' field profile, and the convolution of the imaged flat field and the aperture mode in the image plane will produce a flattened spectral response. To characterize the flatness of the spectral response we introduce the aspect ratio: $\Delta \lambda_{1 \mathrm{~dB}} / \Delta \lambda_{10 \mathrm{~dB}}$, where $\Delta \lambda_{1 \mathrm{~dB}}$ and $\Delta \lambda_{10 \mathrm{~dB}}$ are the $1 \mathrm{~dB}$ and $10 \mathrm{~dB}$ band width of the spectral response as shown schematically in Fig. 2. Note that the MMI-AWG intrinsically has a somewhat higher loss than a conventional AWG because of shape mismatch in the image plane of the AWG and the fact that the power in the image is now spread over a wider area to obtain a flat-top wavelength response.

\section{DESIGN OF MMI AND AWG}

All devices were fabricated on $200 \mathrm{~mm}$ SOI wafers having a $220 \mathrm{~nm}$ thick silicon guiding layer on top of a $2 \mu \mathrm{m}$ buried oxide

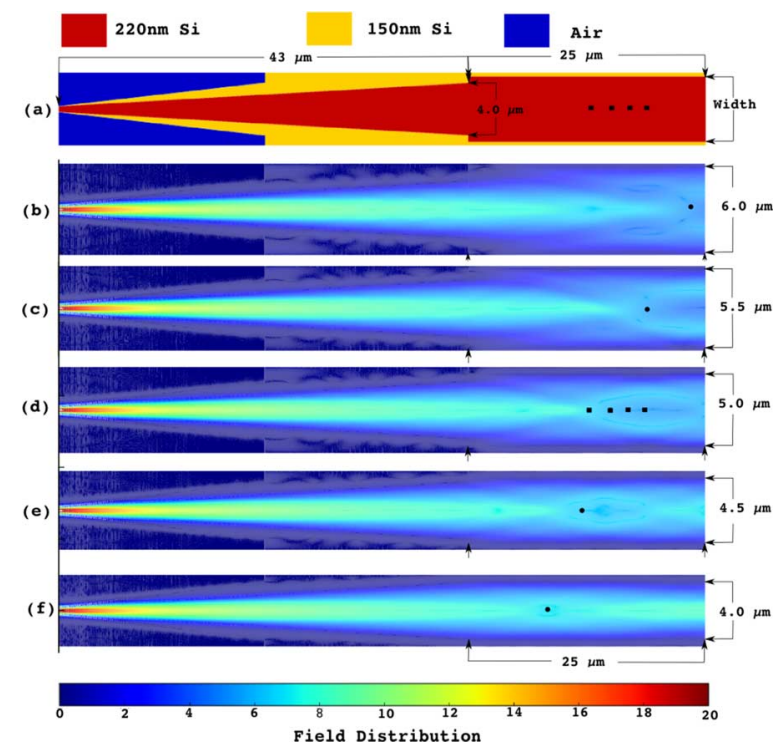

Fig. 3. (a) Geometry of the MMI-aperture used in the simulations and actual design. (b)-(f) Simulated field distribution of the MMI-aperture for different widths. The black circular dots indicate, for a given MMI width, the optimal length for achieving a flat spectral response. The square dots in (d) indicate the positions $13 \mu \mathrm{m}, 15 \mu \mathrm{m}, 17 \mu \mathrm{m}$ and $19 \mu \mathrm{m}$ of the $5 \mu \mathrm{m}$ wide MMI for which Fig. 5 shows the field distribution.

layer. The patterns were defined using $193 \mathrm{~nm}$ deep UV lithography. A double etch process was used: $220 \mathrm{~nm}$ deep trenches define the high contrast photonic wires (further referred to as the deep etch) and a $70 \mathrm{~nm}$ etch defined grating couplers and lower contrast waveguides in the star coupler regions (further referred to as shallow etch). See [10] for further fabrication details. The shallow etch was used for defining all star coupler apertures, including the MMIs, to reduce reflections at the transitions. The array waveguides were tapered to $2 \mu \mathrm{m}$ at the star coupler interface and separated by $0.2 \mu \mathrm{m}$. Also the output apertures are $2 \mu \mathrm{m}$ wide and through a $25 \mu \mathrm{m}$ long adiabatic taper connected to a $450 \mathrm{~nm}$ wide photonic wire. The input apertures of the MMI-AWGs consist of a $42 \mu \mathrm{m}$ long taper having a width of $4 \mu \mathrm{m}$ coupled to an MMI with length and width varied as discussed below. Both the MMI and the taper are shallowly etched.

As discussed above, through the MMI at the input of the AWG we can tailor the image of the input field for an optimized overall response. The MMI aperture needs to be about twice as broad as the output aperture to be able to create an image which is sufficiently flat. For narrower MMIs, the profile will be too narrow, for broader MMIs, the profile will have a significant dip in the middle. It also depends strongly on the dispersion of the waveguide array, i.e., the lateral shift of the spot as function of the wavelength. Fig. 3 shows the simulated field distribution for $4.0 \mu \mathrm{m}, 4.5 \mu \mathrm{m}, 5.0 \mu \mathrm{m}, 5.5 \mu \mathrm{m}$ and 6.0 $\mu \mathrm{m}$ wide and up-to $25.0 \mu \mathrm{m}$ long MMIs. The black circular dots in the figure indicate the optimal length to achieve a flat spectral response. (The simulation method will be explained in Section IV.) When used in an AWG the MMI should interface at this point with the FPR. A line scan of the field profile at these positions is shown in Fig. 4 (as a function of width) and in Fig. 5 (as a function of length). From this figure it is obvious that the convolution of the output waveguide field profile (dash-dot line) with the input field profile of the $4.0 \mu \mathrm{m}$ wide MMI-aperture will produce the lowest loss but will have a spectral response 


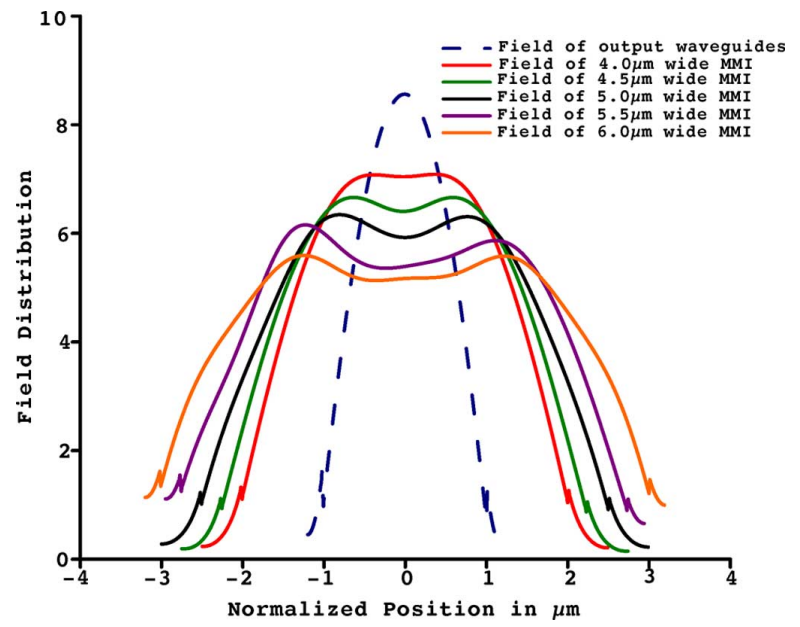

Fig. 4. Simulated field distribution of $4.0 \mu \mathrm{m}, 4.5 \mu \mathrm{m}, 5.0 \mu \mathrm{m}, 5.5 \mu \mathrm{m}$ and $6.0 \mu \mathrm{m}$ wide MMI-apertures for optimal length.

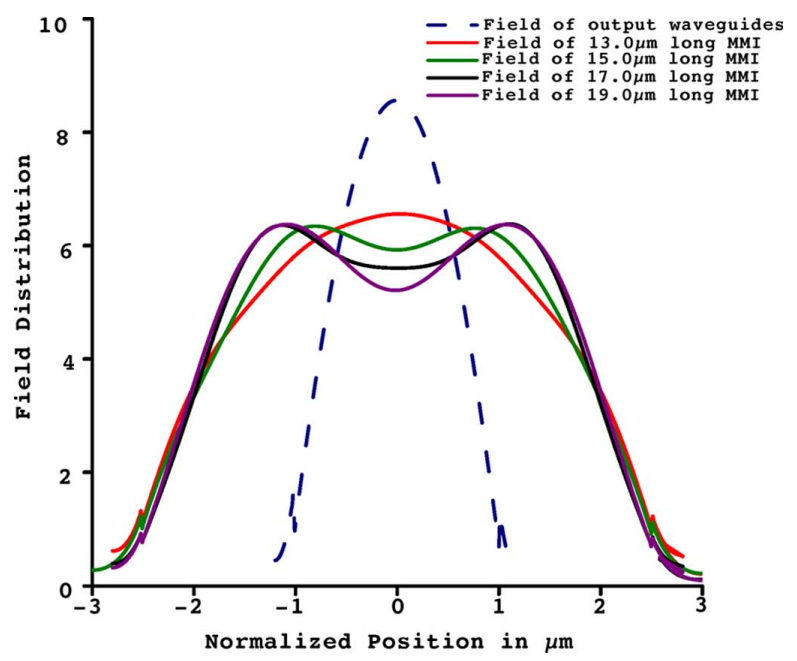

Fig. 5. Field distribution at the end of the MMI for different lengths.

with narrower, more rounded top. The $4.5 \mu \mathrm{m}$ and $5.0 \mu \mathrm{m}$ wide MMI-apertures (close to twice the width of the output aperture) are the best compromise for achieving a low loss and flat spectral response. The loss will increase significantly for further increments in width.

The flatness of the spectral response depends on the length of the MMI-aperture as well. To illustrate this we use a $5.0 \mu \mathrm{m}$ wide MMI-aperture. Fig. 3(d) shows the field as it propagates through the $5.0 \mu \mathrm{m}$ wide and $25.0 \mu \mathrm{m}$ long MMI-aperture. In Fig. 5 we can see how the field distribution changes from a Gaussian-like to a twofold image with a center dip, by varying the length of the MMI from $13 \mu \mathrm{m}$ to $19 \mu \mathrm{m}$ (the width of the MMI in this example was fixed to $5 \mu \mathrm{m}$ ). At a certain point along the length of the MMI, we get a field profile with a flat region in the center. The convolution of this flat image field and the mode in the image plane will produce a flattened spectral response. The width of the flat region in the spectral response depends on the width of the input field at the entrance of the first free propagation region from the MMI-aperture.

Also the number of waveguides in the dispersive array will strongly influence the transfer characteristics of the device. The acceptance angle of the array is fixed by the apertures used at the input and output planes of the star couplers. Hence, if

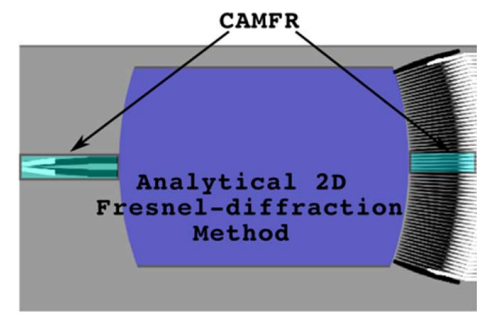

(a)

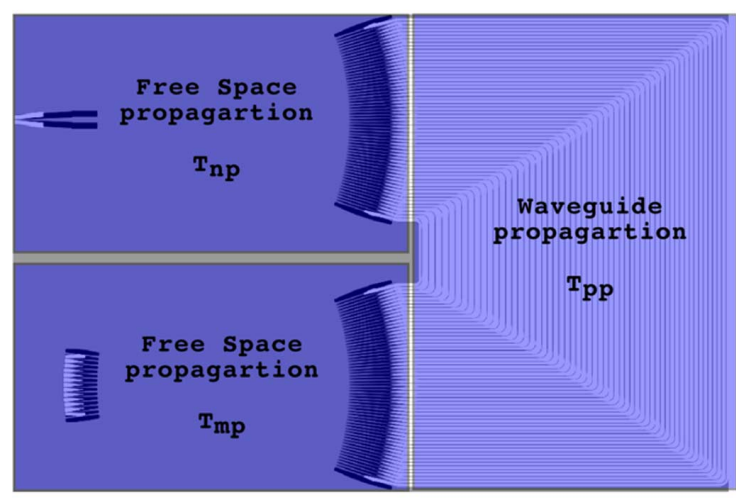

(b)

Fig. 6. (a) Hybrid-analytical model for star-coupler simulation. (b) T-matrix approach for AWG.

we increase the number of waveguides in the array, we will also have to increase the focal length of the FPR. This in turn, for a given shift in wavelength, will increase the lateral shift in the image plane. Also, the increase in number of waveguides itself will improve the image sharpness. Therefore, the $1 \mathrm{~dB}$ normalized passband width $\left(\Delta \lambda_{1 \mathrm{~dB}} / \Delta \lambda\right)$ and the $10 \mathrm{~dB}$ normalized passband width $\left(\Delta \lambda_{10 \mathrm{~dB}} / \Delta \lambda\right.$, where $\Delta \lambda$ is the channel spacing) will be smaller and the neighboring channel crosstalk will be lower. On the other hand, a higher number of waveguides will introduce more phase errors, resulting in a larger overall crosstalk floor. As will be demonstrated in Section V, a good compromise for the number of waveguides to keep both the steepness of the pass band and the crosstalk floor within limits is to use between 2.5 and 4 times the number of wavelength channels in one FSR. However, the passband aspect ratio, i.e., the ratio between the $1 \mathrm{~dB}$ and $10 \mathrm{~dB}$ normalized passband width $\left(\Delta \lambda_{1 \mathrm{~dB}} / \Delta \lambda_{10 \mathrm{~dB}}\right)$ is largely independent of the number of waveguides, but almost fully determined by the overlap of the image with the output aperture mode. To map the influence of the number of waveguides, we designed AWGs with 44, 47, 50, 53 and 56 waveguides.

\section{AWG MODEL}

To simulate the AWG we developed a semi-analytical model integrated with our design software (IPKISS). IPKISS is a modular framework: the components are based on parametric cells, which can be reused, modified and portable between the technologies: the technology informations contains the default design rules and fabrication informations based on the foundry [11]. Our model simulates the transmission matrices (T-matrix) of all parts of the AWG using the most suitable method [12]. In our approach we divided the AWG in three parts: two star-couplers (FPR) and the waveguide array. Fig. 6(b) illustrates our simulation approach for an AWG. 


\section{A. Free Space Propagation Region}

For the star coupler simulation we developed a hybrid-analytical model. As shown in Fig. 6(a) the star-couplers are again split in separate parts: the input apertures, the output apertures and the free space region. To obtain the field profile, the apertures are simulated numerically using eigenmode propagation (CAMFR [13]). Fig. 3(a) shows the geometry used for the CAMFR simulation and Fig. 3(d) shows the field distribution of an MMI aperture simulated by CAMFR. Alternatively, we can use $2 \mathrm{D}$ or $3 \mathrm{D}$ FDTD to simulate the aperture. The calculated field-profile is used as the input for the free-space propagation region, which is solved analytically using a $2 \mathrm{D}$ Fresnel-diffraction scheme. The transmission from the input aperture to the output aperture of the star-coupler is obtained by calculating the overlap between the field-profile obtained from the free space propagation with the field-profile of the output aperture. Finally by using this process repetitively we can build the T-matrix of a star coupler with multiple inputs and outputs. The coupling between two successive apertures is not taken into account. From FDTD simulations we observed that this coupling is negligible small for our devices.

\section{B. Waveguide Array}

The transmission of the waveguides is given by $T=\exp (j$. $\left.\left(2 \pi \cdot \eta_{\mathrm{eff}}(\lambda) / \lambda\right) \cdot L-\alpha_{\mathrm{loss}} \cdot L+j \cdot \sigma_{\text {phase }}(\lambda) \cdot L^{1 / 2}\right)$, where $\eta_{\text {eff }}(\lambda)$ is the effective index, $L$ is the length of the waveguide, $\lambda$ is the operating wavelength, $\alpha_{\text {loss }}$ is introduced to take into account the propagation loss and the last term is used to simulate phase errors due to small variations in waveguide width. To quantify the phase error we assumed that the variations on each sidewall to be stationary and uncorrelated [14]. Thus, the phase error deviation can be described as $\sigma_{\text {phase }}(\lambda)=\left(L_{c}\right.$. $\left.(\delta \beta(\lambda) / \delta \omega)^{2} \cdot \sigma_{\text {width }}^{2}\right)^{1 / 2}$, where $L_{c}$ is correlation length along which the sidewall approximately keeps a constant lateral position, $\delta \beta(\lambda) / \delta \omega$ is the variation of propagation constant with the variation of the waveguide width for the operating wavelength $\lambda$, and $\sigma_{\text {width }}^{2}$ is the variance of the waveguide width deviation. This will introduce an additional phase factor in the transmission of the waveguides depending on the length of the waveguide. As the distribution of the sidewall roughness over a waveguide is random, we used Monte Carlo methods to estimate the phase error variation by running the simulation for 100 samples.

\section{T-Matrix}

We consider an AWG with $n$ input channels, $m$ output channels and $p$ arms. Then the T-matrix of the input star coupler is $T_{n p}$, the T-matrix of the output star coupler is $T_{m p}$ and the T-matrix of the array waveguides is $T_{p p}$. So the overall T-matrix $\left(T_{m n}\right)$ of the AWG is calculated as $T_{m p} \cdot T_{p p} \cdot T_{n p}^{T}$.

\section{RESUlts AND Discussion}

To characterize the device grating couplers are integrated with the input and output channels of the AWG's. These have a coupling efficiency with standard single mode fiber of nearly $30 \%$ [15]. To eliminate the effect of the grating coupler from the AWG's spectral response we subtract the transmission of the AWG from the transmission of a reference waveguide having identical grating couplers. In this section first we sepa-

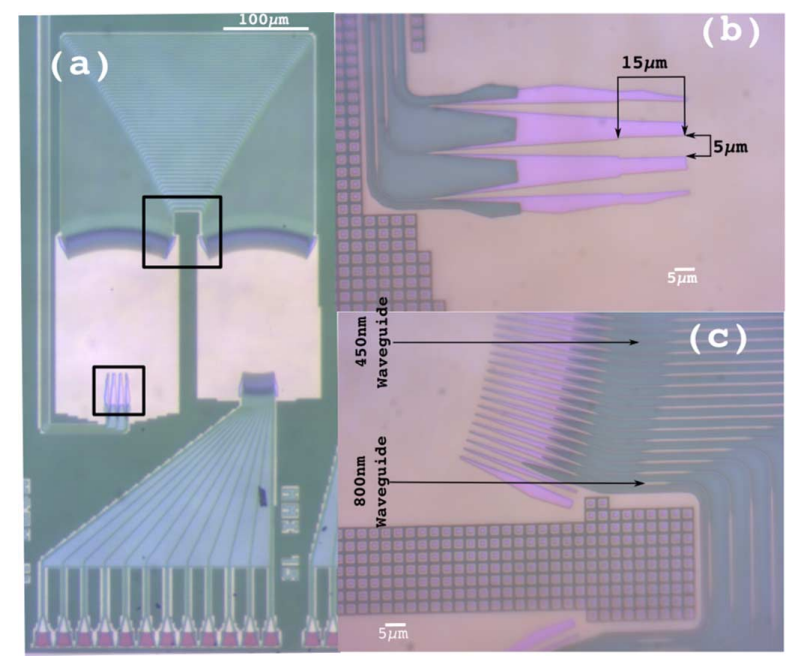

Fig. 7. (a) Microscopic images of the fabricated $12 \times 400 \mathrm{GHz}$ MMI-AWG. (b) Microscopic images of the fabricated mmi aperture. (c) Microscopic images of the fabricated waveguide array.

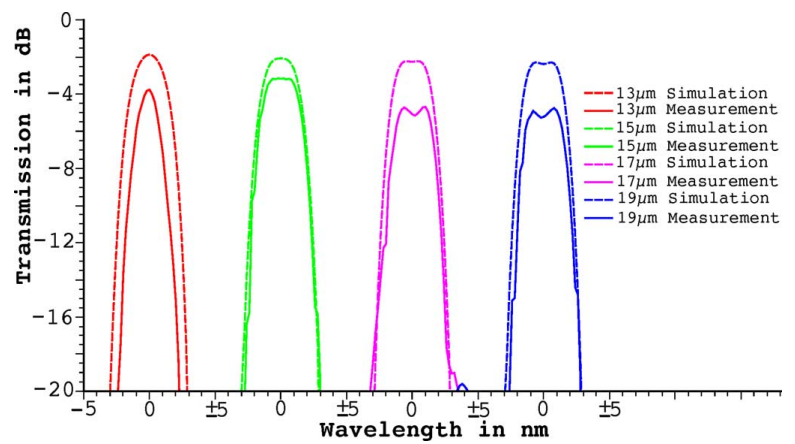

Fig. 8. Simulated spectral response of $400 \mathrm{GHz}$ AWG with variation of MMI lengths.

rately describe the effect of the MMI length and the effect of the number of waveguides used in the waveguide array on the AWG spectral response. Next we discuss the characterization of the MMI-AWG optimized based on the previous results. Fig. 7 shows the fabricated MMI-AWGs under characterization.

\section{A. Effect of MMI Length}

The simulated and experimental spectral response of MMIAWGs with four different MMI lengths $(13 \mu \mathrm{m}, 15 \mu \mathrm{m}, 17 \mu \mathrm{m}$ and $19 \mu \mathrm{m}$ ) is shown in Fig. 8. The width of the MMIs was equal to $5 \mu \mathrm{m}$ in each case. We observe that there is a significant qualitative correspondence: the $13 \mu \mathrm{m}$ MMIs result in a Gaussian-like response, $15 \mu \mathrm{m}$ MMIs result in a flattop response, $17 \mu \mathrm{m}$ MMIs result in a small dip and $19 \mu \mathrm{m}$ MMIs result in a large dip at the center of the response. The experimental results for the $17 \mu \mathrm{m}$ and $19 \mu \mathrm{m}$ MMI-AWG show a higher center dip than the simulated response, most probably to be explained by a small mismatch in MMI width, waveguide thickness or choice of refractive index used in the simulation. From these results we can conclude that a $15 \mu \mathrm{m}$ long and $5 \mu \mathrm{m}$ wide MMI is suitable for achieving a flat-spectral response.

\section{B. Optimization of Waveguide Array}

The most complex part of the AWG to optimize is the waveguide array. The optical path length difference between two successive waveguides of the array is constant over the array (see 


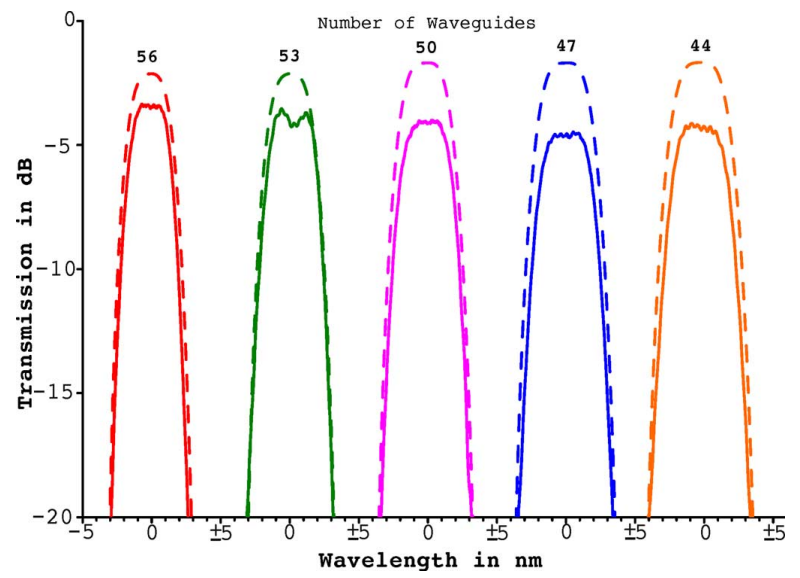

Fig. 9. Simulation and experimental spectral response of the MMI-AWG with variation of the number of waveguides used in the waveguide array. Solid lines indicate the measurement results and dash lines indicate the simulation results.

Section II) and depends on the design parameters (e.g., the desired free spectral range). A key variable is also the number of waveguides to be used in the array, which influences the steepness of the pass band and the crosstalk floor. As discussed above, a good compromise for the number of waveguides is to use between 2.5 and 4 times the number of wavelength channels in one FSR (see Section III). To map the influence of the number of waveguides, we designed $12 \times 400 \mathrm{GHz}$ AWGs with 44, 47, 50,53 and 56 waveguides in the array, whereby an MMI-aperture of $5 \mu \mathrm{m}$ wide and $15 \mu \mathrm{m}$ long was used as the input for the first star coupler. Fig. 9 shows the simulated and experimental spectral response for these devices. Both in the simulated and the experimental results we observe that the pass band width increases as the number of waveguides decreases, but that the flatness of the response is not affected.

1) Bandwidth and Aspect Ratio: The simulated and experimental $1 \mathrm{~dB}$ and $10 \mathrm{~dB}$ normalized passband widths of AWG's with increasing number of array waveguide are shown in Fig. 10 (a). The $1 \mathrm{~dB}$ and $10 \mathrm{~dB}$ normalized passband width decreases with an increasing number of waveguides in the array, both for conventional and for MMI-AWG's. For the MMI-AWG the experimental result matches the simulation very well. In Fig. 10(b) we can see that the aspect ratio of the conventional AWGs is much lower than that of the MMI-AWGs: simulated values for the aspect ratio of conventional AWGs and MMI-AWGs are 0.33 and 0.49 respectively. These values are nearly independent of the number of waveguides in the array. The measurement result of the aspect ratio for MMI-AWGs matches well with the simulation. This shows in a quantitative way that we get a much flatter passband response from the MMI-AWG.

2) Crosstalk: The crosstalk is mainly dependent on the number of waveguides used in the waveguide array. From Fig. 10(a) we can observe that with 44 waveguides in the array the $1 \mathrm{~dB}$ bandwidth is nearly equal to the channel spacing of the AWG (means normalized passband width equal to one), implying that the neighboring channel crosstalk will be high. For a higher number of waveguides the neighboring channel crosstalk decreases. Fig. 11 shows that the experimental neighboring channel crosstalk follows the same trend as the simulated results. As the number of waveguides increases the effect of phase errors also increase however. The phase errors are mostly dependent on the side wall roughness and thickness

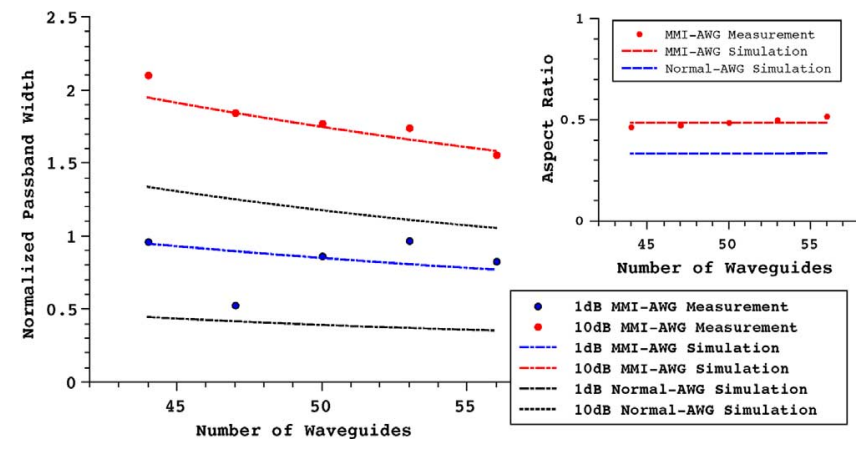

Fig. 10. (a) Passband width (passband width is normalized to the channel spacing) vs. number of waveguides used in the waveguide array of the MMI-AWG. (b) Comparison of the aspect ratio between conventional-AWG and MMI-AWG.

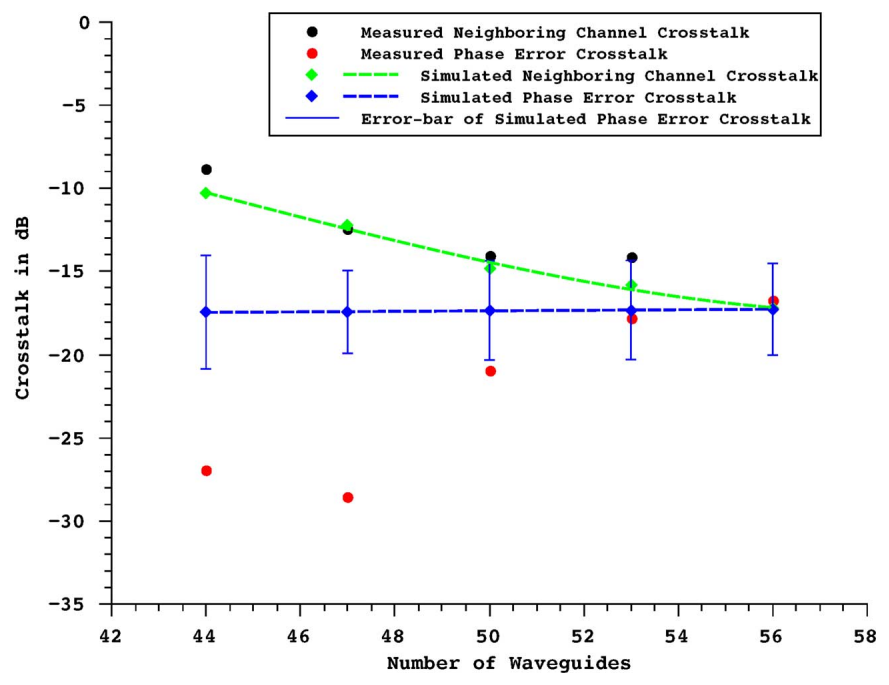

Fig. 11. Crosstalk vs. number of waveguides used in the waveguide array of the MMI-AWG.

variations of the waveguides in the waveguide array. In Fig. 11 we can observe that for lower numbers of waveguides in the AWG, the effect of phase errors decreases. For 56 waveguides the neighboring channel crosstalk and the crosstalk floor are at the same level. When further increasing the number of waveguides, the neighboring channel crosstalk is hidden in the crosstalk floor. In Fig. 11 we can also observe that the experimental phase errors do not match the simulated phase errors. The reason behind this discrepancy is in the phase error model, which is based on a one-dimensional approximation while in reality the thickness variation and the inclined sidewall of the waveguide have an important impact on the phase error, and phase errors between waveguides might be correlated.

\section{Optimized Spectral Response}

From the previous results we can conclude that the $12 \times 400 \mathrm{GHz}$ MMI-AWG with 56 waveguides and a $15 \mu \mathrm{m}$ long and $5 \mu \mathrm{m}$ wide MMI as the input aperture presents a good compromise in achieving a flat response with minimal loss and good crosstalk level. Using our AWG model (see Section IV) we simulated this device now in more detail. Fig. 12 shows that the device is expected to exhibit a central channel loss of $-2.07 \mathrm{~dB}$, an insertion loss non-uniformity of $0.8 \mathrm{~dB}$ and a crosstalk level of $19.5 \mathrm{~dB}$ [16], which is defined by the neighbor channels. Experimentally we find (Fig. 13) a central channel 


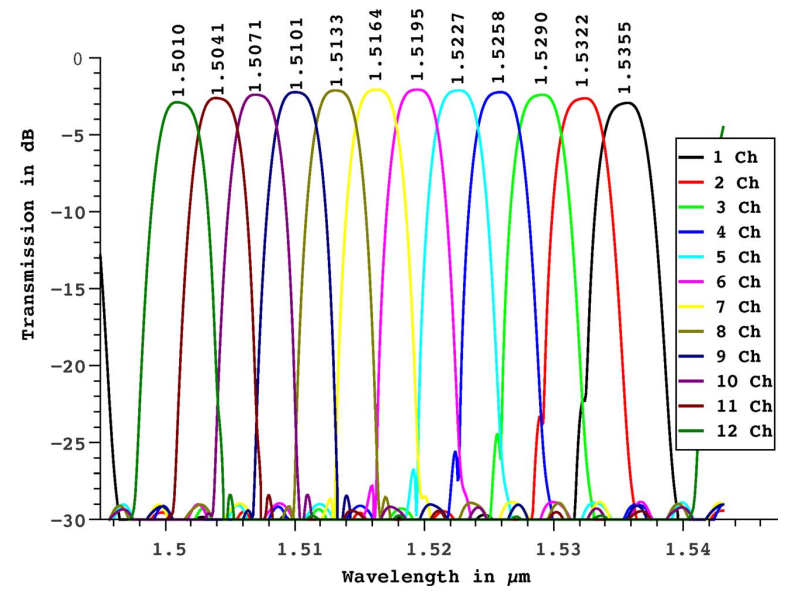

Fig. 12. Simulation result of $12 \times 400 \mathrm{GHz}$ MMI-AWG.

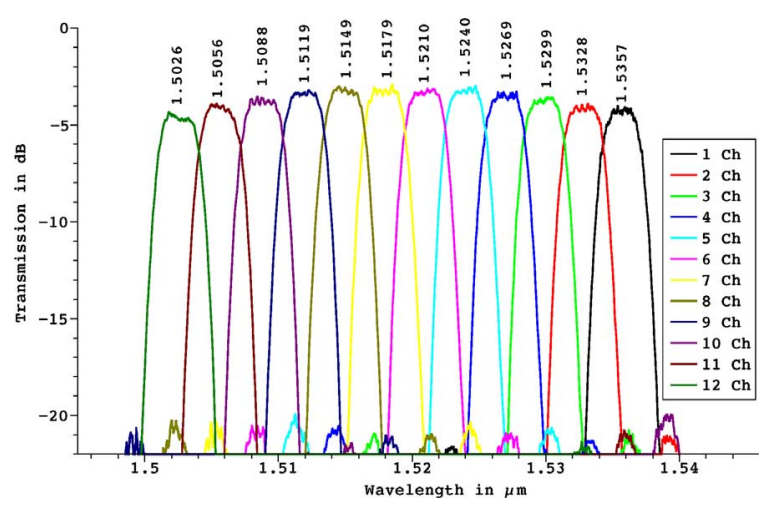

Fig. 13. Measurement result of $12 \times 400 \mathrm{GHz}$ MMI-AWG.

loss of $-3.29 \mathrm{~dB}$, an insertion loss non-uniformity of $1.55 \mathrm{~dB}$ and a crosstalk of $17.0 \mathrm{~dB}$ [16], which is defined by the phase error level. In both simulation and experiment we see that not all channels have a perfect flat-top response, but are slightly asymmetric: the left side channels have left side increments and the right side channels have right side increments. This is an effect of imaging aberrations for the outer channels [17]. As expected the MMI-AWG also has a somewhat higher loss than a conventional AWG with the same design parameters because of the shape mismatch in the image plane of the AWG and the fact that the power in the image is now spread over a wider area to obtain a flat-top wavelength response. The difference in center channel loss between simulation and experiment result is due to the waveguide propagation loss, the slab propagation loss and the propagation angle of the aperture mismatch between the designed and the fabricated AWGs. The dispersion mismatch and the aperture field profile mismatch between the designed and the fabricated waveguide is the reason behind the mismatch of insertion loss non-uniformity. One of the main concerns for this type of device is the non-uniformity of bandwidth and channel spacing over the different channels due to the dispersion mismatch between the designed and the fabricated waveguide. Fig. 14 shows the fiber to fiber measurement of the device and the reference waveguide. As we can see from Fig. 14 that the envelope of the device spectrum follow the envelope of the reference waveguide spectrum. If we change the coupling method this envelope of the reference waveguide as well as the envelope of the device spectrum will change. We observe that

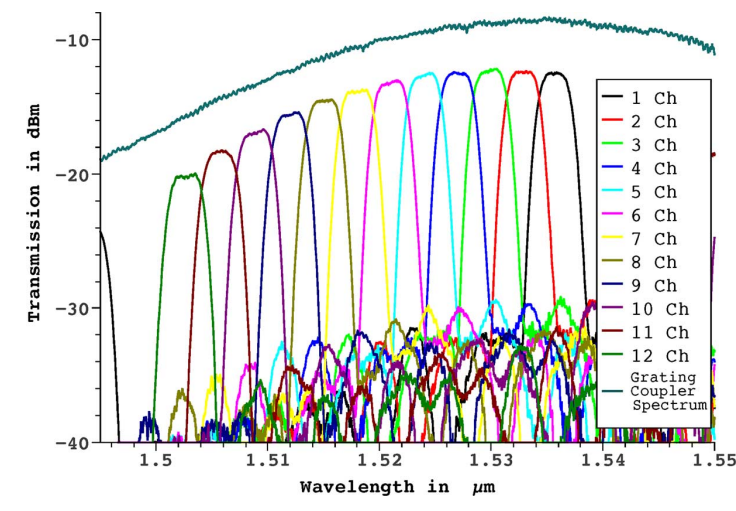

Fig. 14. Fiber to fiber measurement Result of $12 \times 400 \mathrm{GHz}$ MMI-AWG and the reference waveguide.

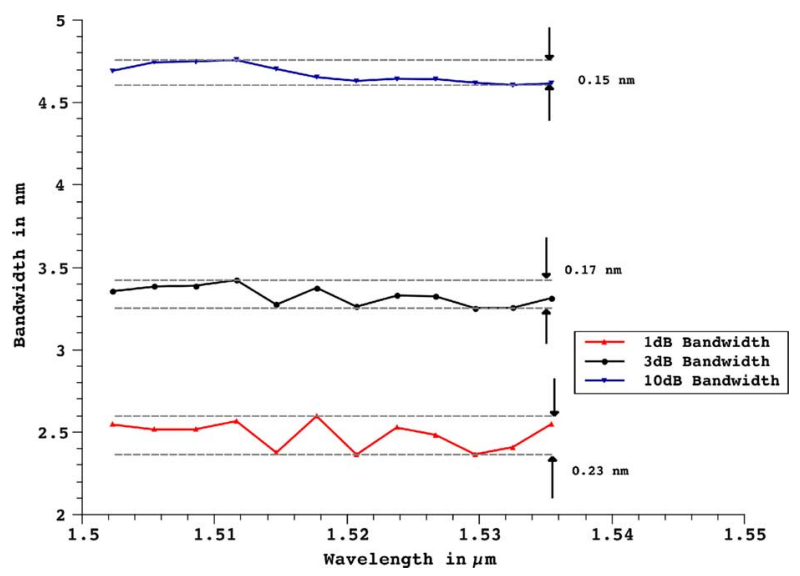

Fig. 15. $1 \mathrm{~dB}, 3 \mathrm{~dB}$ and $10 \mathrm{~dB}$ bandwidth for $12 \times 400 \mathrm{GHz}$ MMI-AWG. Associated non-uniformity is indicated in the graph.

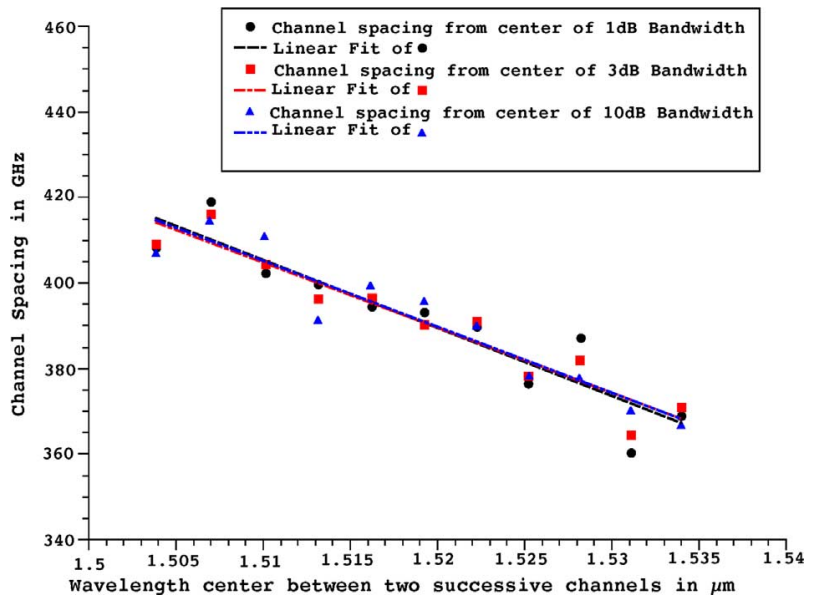

Fig. 16. Channel spacing variation of the $12 \times 400 \mathrm{GHz}$ MMI-AWG.

for this device (shown in Fig. 15) the non-uniformity of the $1 \mathrm{~dB}$, $3 \mathrm{~dB}$ and $10 \mathrm{~dB}$ bandwidths is $0.23 \mathrm{~nm}, 0.17 \mathrm{~nm}$ and $0.15 \mathrm{~nm}$ respectively. Fig. 16 shows that the channel spacing decreases as the wavelength increases although the device was designed for a constant channel spacing of $400 \mathrm{GHz}$, taking into account first-order dispersion. The deviation between experimental results and design value can be explained by the mismatch in the dispersion relation and waveguide thickness used in our model compared to the actually fabricated waveguide. The average channel spacing obtained from Fig. 16 is $391 \mathrm{GHz}$. 


\section{CONCLUSION}

A compact 12-channel flat-top arrayed waveguide grating (AWG) on silicon-on-insulator (SOI) was presented. The device size is $560 \times 350 \mu \mathrm{m}^{2}$. Center channel loss is $-3.29 \mathrm{~dB}$, crosstalk is $17 \mathrm{~dB}$, average channel spacing is $391 \mathrm{GHz}$, aspect ratio is 0.52 and imaging error is low. The experimental results match well with simulation results. The effect of the MMI length on the spectral response of the AWG is demonstrated in detail both through simulation as by experimental results. The aspect ratio of the channel response of the MMI-AWGs is considerably improved compared to that of a conventional AWG. The neighboring channel crosstalk depends on the number of waveguides and also matches well with the simulations. The non-uniformity of the bandwidths is limited and given by $0.23 \mathrm{~nm}, 0.17 \mathrm{~nm}$ and $0.15 \mathrm{~nm}$ for the $1 \mathrm{~dB}, 3 \mathrm{~dB}$ and $10 \mathrm{~dB}$ bandwidth respectively. The average channel spacing obtained from the experiment is $391 \mathrm{GHz}$. These results demonstrate that we are able to design and fabricate compact silicon flat passband AWGs with predictable spectral response.

\section{ACKNOWLEDGMENT}

The experimental AWG devices were fabricated through ePIXfab.

\section{REFERENCES}

[1] M. Smit and C. Van Dam, "PHASAR-based WDM-devices: Principles, design and applications," IEEE J. Sel. Top. Quantum Electron., vol. 2, no. 2, pp. 236-250, 1996.

[2] J. Soole, M. Amersfoort, H. LeBlanc, N. Andreadakis, A. Rajhel, C. Caneau, R. Bhat, M. Koza, C. Youtsey, and I. Adesida, "Use of multimode interference couplers to broaden the passband of wavelength-dispersive integrated WDM filters," IEEE Photon. Technol. Lett., vol. 8, no. 10 , pp. $1340-1342,1996$.

[3] R. Adar, C. Henry, C. Dragone, R. Kistler, and M. Milbrodt, "Broadband array multiplexers made with silica waveguides on silicon," $J$. Lightw. Technol., vol. 11, no. 2, pp. 212-219, 1993.

[4] M. Zirngibl, C. Dragone, and C. Joyner, "Demonstration of a $15 \times 15$ arrayed waveguide multiplexer on InP," IEEE Photon. Technol. Lett., vol. 4, no. 11, pp. 1250-1253, 1992.

[5] Y. Vlasov et al., "Losses in single-mode silicon-on-insulator strip waveguides and bends," Opt. Express, vol. 12, no. 8, pp. 1622-1631, 2004.

[6] S. Selvaraja, W. Bogaerts, and D. Van Thourhout, "Loss reduction in silicon nanophotonic waveguide micro-bends through etch profile improvement," Opt. Commun., vol. 284, no. 8, pp. 2141-2144, 2011.

[7] Q. Fang, T. Liow, J. Song, K. Ang, M. Yu, G. Lo, and D. Kwong, "WDM multi-channel silicon photonic receiver with 320 Gbps data transmission capability," Opt. Express, vol. 18, no. 5, pp. 5106-5113, 2010.

[8] K. Sasaki, F. Ohno, A. Motegi, and T. Baba, "Arrayed waveguide grating of $70 \times 60 \mu \mathrm{m}^{2}$ size based on Si photonic wire waveguides," Electron Lett., vol. 41, no. 14, pp. 801-802, 2005.

[9] W. Bogaerts, S. Selvaraja, P. Dumon, J. Brouckaert, K. De Vos, D. Van Thourhout, and R. Baets, "Silicon-on-insulator spectral filters fabricated with CMOS technology," IEEE J. Sel. Top. Quantum Electron., vol. 16, no. 1, pp. 33-44, 2010.

[10] S. Selvaraja, P. Jaenen, W. Bogaerts, D. Van Thourhout, P. Dumon, and R. Baets, "Fabrication of photonic wire and crystal circuits in silicon-on-insulator using $193 \mathrm{~nm}$ optical lithography," J. Lightw. Technol., vol. 27, no. 18, pp. 4076-4083, 2009.

[11] W. Bogaerts, P. Dumon, E. Lambert, M. Fiers, S. Pathak, and A. Ribeiro, "Ipkiss: A parametric design and simulation framework for silicon photonics," in Proc. 9th IEEE Int. Conf. Group IV Photonics, Aug. 2012, pp. 30-32.

[12] S. Pathak, W. Bogaerts, E. Lambert, P. Dumon, and D. V. Thourhout, "Integrated design and simulation tools for silicon photonic arrayed waveguide gratings," IEEE Photon. Benelux Ch., pp. 41-44, Nov. 2010.
[13] CAvity Modelling FRamework (CAMFR). Dept. Inf. Technol. (INTEC), Ghent Univ., Belgium [Online]. Available: http://camfr. sourceforge.net

[14] T. Goh, S. Suzuki, and A. Sugita, "Estimation of waveguide phase error in silica-based waveguides," J. Lightw. Technol., vol. 15, no. 11, pp. 2107-2113, 1997.

[15] F. Van Laere, T. Claes, J. Schrauwen, S. Scheerlinck, W. Bogaerts, D. Taillaert, L. O'Faolain, D. Van Thourhout, and R. Baets, "Compact focusing grating couplers for silicon-on-insulator integrated circuits," IEEE Photon. Technol. Lett., vol. 19, no. 23, pp. 1919-1921, 2007.

[16] S. Pathak, E. Lambert, P. Dumon, D. Van Thourhout, and W. Bogaerts, "Compact SOI-based AWG with flattened spectral response using a MMI," in Proc. 8th IEEE Int. Conf. Group IV Photonics, Sep. 2011, pp. $45-47$.

[17] A. Klekamp and R. Münzer, "Calculation of imaging errors of AWG," J. Lightw. Technol., vol. 21, no. 9, pp. 1978-1986, 2003.

Shibnath Pathak received the M.Sc. degree in physics from the Indian Institute of Technology Madras, Chennai, India, in 2009. He is currently working toward the Ph.D. degree at the Photonic Research Group, Ghent University-Interuniversity Microelectronics Center (IMEC), Ghent, Belgium.

His current research interests include the modeling, design, characterization of the grating based wavelength-division multiplexing devices and optical interconnects.

Mr. Pathak is a Student Member of the IEEE Photonics Society.

Michael Vanslembrouck received the Master degree in computer sciences engineering from Ghent University, Ghent, Belgium, in 1996.

$\mathrm{He}$ is currently a computer engineer at the Photonic Research Group, Ghent University-Interuniversity Microelectronics Center (IMEC), Ghent, Belgium. His current work focuses on the automation of photonic measurements on die and wafer scale.

Pieter Dumon received the Master degree in electronics engineering in 2002 and the Ph.D. degree in 2007, both from Ghent University, Belgium.

$\mathrm{He}$ is currently with imec, Ghent University, as a design engineer for silicon photonic ICs and manager of ePIXfab. He specializes in the modeling and design of photonic components and circuits and the intimate relation between design and fabrication technology and process variability.

Dr. Dumon is a member of the IEEE Photonics Society.

Dries Van Thourhout received the degree in physical engineering and the Ph.D. degree in applied physics from Ghent University, Ghent, Belgium, in 1995 and 2000, respectively.

From October 2000 to September 2002, he was with Lucent Technologies, Bell Laboratories, Murray Hill, NJ, where he was involved in the designing, processing, and characterization of InP/InGaAsP monolithically integrated devices. In October 2002, he joined the Department of Information Technology, Ghent University, where he is currently a member of the permanent staff of the Photonics Group. He is a Lecturer or Co-Lecturer for four courses within the Ghent University Master in Photonics program (Microphotonics, Advanced Photonics Laboratory, Photonic Semiconductor Components and Technology). $\mathrm{He}$ is also coordinating the cleanroom activities of the research group. He is also engaged for the development of new fabrication processes for photonic devices, e.g., based on focused ion-beam etching and die-to-wafer bonding. He holds four patents. He has authored and coauthored more than 120 journal papers and invited papers at several major conferences. His research interests include the design, fabrication, and characterization of integrated photonic devices, silicon nanophotonic devices, and heterogeneous integration of InP-on-silicon.

Dr. Van Thourhout is a member of IEEE Laser and Electrooptics Society and an Associate Editor for IEEE Photonics Technology Letters.

Wim Bogaerts completed his studies in engineering (applied physics) at Ghent University, Belgium, in 1998 and joined the Department of Information Technology (INTEC) at both Ghent University and the Interuniversity Microelectronics Center (IMEC) where he received the Ph.D. degree in 2004. In the Photonics Research Group he specialized in the modeling, design and fabrication of nanophotonic components.

He is currently a Professor in the field of silicon photonics at Ghent University, and coordinates the activities between imec and Ghent University in silicon photonics design.

Prof. Bogaerts is a member of the IEEE Photonics Society, the Optical Society of America (OSA) and SPIE. 